\title{
MORSE-NOVIKOV THEORY, HEEGAARD SPLITTINGS, AND CLOSED ORBITS OF GRADIENT FLOWS
}

\author{
H. GODA, H. MATSUDA, AND A. PAJITNOV
}

\begin{abstract}
The work of Donaldson and Mark made the structure of the SeibergWitten invariant of 3-manifolds clear. It corresponds to certain torsion type invariants counting flow lines and closed orbits of a gradient flow of a circle-valued Morse map on a 3-manifold. In the paper, these invariants are studied by using the Morse-Novikov theory and Heegaard splitting for sutured manifolds, and detailed computations are made for knot complements.
\end{abstract}

\section{§1. INTRODUCTION}

Let $K \subset S^{3}$ be an oriented knot; we put $C_{K}=S^{3}-K$. The canonical cohomology class $\xi \in H^{1}\left(C_{K}\right)=\left[C_{K}, S^{1}\right]$ can be represented by a Morse map $f: C_{K} \rightarrow S^{1}$. In this paper we study the dynamics of the gradient flow of $f$.

In [16, Milnor pointed out a relationship between the Reidemeister torsion and dynamical zeta functions. His theorem applies to fibered knots, that is to the case where we can choose the map $f$ without critical points. The theorem implies in particular that the Alexander polynomial of any fibered knot in $S^{3}$ is essentially the same as the Lefschetz zeta function of the monodromy map of the fibration $f$. The periodic points of the monodromy map correspond to the closed orbits of the gradient flow of the fibration $C_{K} \rightarrow S^{1}$; thus, Milnor's theorem establishes a relationship between the dynamics of this gradient flow and the Alexander polynomial of the knot.

When the knot $K$ is not fibered, the Morse map $f$ necessarily has critical points. Milnor's formula is no more valid, however it can be generalized to this case at the cost of adding a correction term, as it was discovered by Hutchings and Lee [11, 12. This correction term is essentially the torsion of the Novikov complex associated with the circle-valued Morse map $f$ (see [18, 20]). This complex is an analog of the Morse complex for the circle-valued case, and is obtained through counting the flow lines of the gradient joining the critical points of the map.

The torsion of the Novikov complex and the Lefschetz zeta function are in general very difficult to compute due to the complexity of the transversal gradient flows used in the construction of the Novikov complex. In the paper 14, Mark introduced a new class of gradient flows for circle-valued Morse maps (symmetric flows), which are not transversal but, somewhat unexpectedly, the Morse-Novikov theory can be extended to this case. He used these flows to give yet another proof of the Meng-Taubes theorem (see the original paper of Meng and Taubes [15] and the later papers of Turaev [24] and Donaldson 2 for alternative proofs of that theorem).

2010 Mathematics Subject Classification. Primary 57M27.

Key words and phrases. Oriented knot, sutured manifold, Morse map, Novikov complex, halftransversal gradients, Lefschetz zeta function.

The first and second authors were partially supported by Grant-in-Aid for Scientific Research (No. 21540071 and No. 20740041), Ministry of Education, Science, Sports and Technology, Japan. 
The symmetric flows have a simple geometric structure allowing us to carry a large part of the Morse-Novikov theory over to this setting, and on the other hand to perform explicit computations with these flows. This is our main aim in the present paper. We begin with studying the geometric properties of symmetric gradients (we work actually with a slightly wider class of vector fields called half-transversal gradients), and establish the basic theorem of the Morse-Novikov theory for this class of flows. This theorem is valid in a more general context than Mark's results, and we believe that our proof is simpler.

Then we proceed to a detailed study of the geometry of the Morse map $f$. In the case where $f$ is a fibration, the first return map from a regular fiber to itself is a diffeomorphism, called the monodromy of the fibration; this is the basic notion that helps to understand the dynamics of the gradient flow. We generalize this notion to the case where $f$ has critical points. Our monodromy is a diffeomorphism of two surfaces constructed from a Heegaard splitting for the complement of a knot [6] (we recall the basic notions of the theory of Heegaard splittings in \$5). This diffeomorphism depends on the choice of the gradient, however it can be computed efficiently in particular cases, which leads to the computation of the Lefschetz zeta function of certain symmetric gradients for the twist knots and the pretzel knot of type $(5,5,5)$. The monodromy enables us also to compute the determinant of the boundary operator in the Novikov complex for the case of these knots (the so-called Novikov torsion).

The dynamics of the gradient flows of circle-valued Morse maps is closely related to the Seiberg-Witten invariants of 3-manifolds. Meng and Taubes [15] showed that the Seiberg-Witten invariant of any closed 3-manifold $M$ with $b_{1}(M) \geq 1$ can be identified with the Milnor torsion. Fintushel and Stern [3] proved that for any knot $K$ in $S^{3}$ the Seiberg-Witten invariant of the manifold $M \times S^{1}$, where $M$ is the result of the zerosurgery on $K$, equals the Alexander polynomial of $K$ multiplied by a certain standard factor. In [2], Donaldson gave a new proof of the Meng-Taubes theorem by applying ideas from topological quantum field theory. These TQFTs were used by Mark to prove a conjecture of Hutchings-Lee concerning the relationship of the Seiberg-Witten invariants with the Novikov torsion. Some results of the present paper have been announced in [10].

\section{§. HALF-TRANSVERSAL FLOWS}

Let $f: M \rightarrow S^{1}$ be a Morse function on a closed manifold $M$. The dynamics of the gradient flow of $f$ is best understood when $f$ has no critical points. In this case we choose a regular surface for $f$, and the dynamics of the gradient flow is determined by the first return map of this surface to itself. This map is called the monodromy of the gradient flow. If $f$ has critical points, the situation is much more complicated, because for every transversal $f$-gradient the first return map is defined not everywhere. It turns out however that in the case of 3-dimensional manifolds there is an important class of nontransversal gradient flows for which the first return map determines a selfdiffeomorphism of the level surface. First, we shall give a definition of the corresponding class of gradient flows on cobordisms.

Let $Y$ be a 3-dimensional cobordism; denote $\partial_{-} Y, \partial_{+} Y$ the lower, respectively, the upper boundary of $Y$. Let $\psi: Y \rightarrow[a, b]$ be a Morse map without critical points of indices 0 and 3 . The subset $U_{1}$ of all points $x$ in the upper boundary $\partial_{+} Y$ such that the $(-v)$-trajectory starting at $x$ reaches the lower boundary $\partial_{-} Y$ is open in $\partial_{+} Y$ and the gradient descent determines a diffeomorphism $(-v)^{\rightsquigarrow}: U_{1} \stackrel{\approx}{\rightarrow} U_{0}$ of $U_{1}$ onto an open subset $U_{0} \subset \partial_{-} Y$. 
For two critical points $p, q$ of $f$, we call a flow line of $v$ from $q$ to $p$ an integral curve $\gamma$ of $v$ such that

$$
\lim _{t \rightarrow-\infty} \gamma(t)=q, \quad \lim _{t \rightarrow \infty} \gamma(t)=p
$$

We shall identify two flow lines of $v$ obtained from each other by a reparameterization.

Definition 2.1. A $\psi$-gradient $v$ is called a smooth descent gradient if

(i) the number of critical points of index 1 is equal to the number of critical points of index 2 , and they can be arranged in two sequences

$$
S_{1}(\psi)=\left\{p_{1}, \ldots, p_{k}\right\}, \quad S_{2}(\psi)=\left\{q_{1}, \ldots, q_{k}\right\}
$$

in such a way that for every $i$ there are two flow lines of $v$ joining $q_{i}$ with $p_{i}$ and these $2 k$ flow lines are the only flow lines of $v$;

(ii) the map $(-v)^{\rightsquigarrow}: U_{1} \rightarrow U_{0}$ can be extended to a $C^{\infty}$ map $g: \partial_{+} Y \rightarrow \partial_{-} Y 1$

Now, we return to circle-valued Morse maps.

Let $f: M \rightarrow S^{1}$ be such a map, where $M$ is a 3 -dimensional closed manifold, and let $v$ be an $f$-gradient. Cutting $M$ along a regular surface $S$ of $f$, we obtain a cobordism $Y$, a Morse function $\psi: Y \rightarrow[0,1]$, and a $\psi$-gradient $\bar{v}=v \mid Y$.

Definition 2.2. The $f$-gradient $v$ is said to be half-transversal if there is a regular level surface $S$ such that $\bar{v}=v \mid Y$ is a smooth descent gradient of $\psi=f \mid Y$ and we have the following transversality condition for stable and unstable manifolds:

$$
\mathcal{W}^{s t}(q) \pitchfork \mathcal{W}^{u n}(p)
$$

for every critical points $p, q$ of $f$ with ind $q=2$, ind $p=1$.

It is not difficult to show that the subset of all half-transversal gradients is dense in the set of smooth descent gradients.

Definition 2.3. Let $v$ be a half-transversal gradient for a Morse function $f: M \rightarrow S^{1}$ and $S$ the corresponding level surface of $f$. The first return map for $(-v)$ determines a diffeomorphism of $S$ to itself which will be called the monodromy of the flow generated by $v$, and denoted by $g$.

The notion of a half-transversal gradient, as introduced above, originates from the paper of Mark [14, where the class of symmetric flows was introduced. In our terminology, Mark's symmetric gradient on a cobordism $Y$ is a smooth descent gradient with the following additional restriction: there is an involution $I: Y \rightarrow Y$ swapping the lower and upper boundaries of $Y$ and such that $I_{*}(v)=-v$ and $\psi \circ I$ equals $-\psi$ up to an additive constant. We do not know if the class of smooth descent gradients is really wider than Mark's class of symmetric gradients. However, the existence of the involution $I$ seems restrictive, and we prefer to work with the more general notion of smooth descent gradients.

Now we define the Novikov complex and the Lefschetz zeta function for half-transversal gradient flows. The usual procedure of counting flow lines yields the Novikov incidence coefficient

$$
N\left(q_{i}, p_{j} ; v\right)=\sum_{k \in \mathbf{N}} n_{k}\left(q_{i}, p_{j} ; v\right) t^{k} \in \mathbb{Z}[[t]]
$$

where

$$
n_{k}\left(q_{i}, p_{j} ; v\right)=\sum_{\gamma \in \Gamma_{k}\left(q_{i}, p_{j} ; v\right)} \varepsilon(\gamma)
$$

\footnotetext{
${ }^{1}$ It seems to us that condition i) actually follows from ii), but we cannot prove it at present.
} 
(here $\Gamma_{k}\left(q_{i}, p_{j} ; v\right)$ stands for the set of all flow lines of $(-v)$ joining $q_{i}$ with $p_{j}$, and intersecting $S$ exactly $k$ times, and $\varepsilon(\gamma)$ is the sign attributed to each flow line with respect to the choice of orientations of the 2-dimensional stable manifolds). The Novikov incidence coefficients form a square matrix $D$ with entries in $\mathbb{Z}[[t]]$. The chain complex

$$
0 \longleftarrow \mathcal{N}_{1}^{-} \stackrel{D}{\longleftarrow} \mathcal{N}_{2}^{-} \longleftarrow 0
$$

where $\mathcal{N}_{i}^{-}$is the free $\mathbb{Z}[[t]]$-module generated by the critical points of $f$ of index $i$, is called the positive Novikov complex of the pair $(f, v)$ and is denoted by $\mathcal{N}_{*}^{-}(f, v)$ or simply $\mathcal{N}_{*}^{-}$if no confusion is possible. The chain complex

$$
0 \longleftarrow \mathcal{N}_{1} \stackrel{D}{\longleftarrow} \mathcal{N}_{2} \longleftarrow 0,
$$

where $\mathcal{N}_{i}$ is the free $\mathbb{Z}((t))$-module generated by the critical points of $f$ of index $i$, is called the Novikov complex of the pair $(f, v)$ and is denoted by $\mathcal{N}_{*}(f, v)$ or simply $\mathcal{N}_{*}$ if no confusion is possible. The first of the two chain complexes above is more convenient in computations; however, only the homotopy type of the second is a homotopy invariant of the map $f: M \rightarrow S^{1}$ (see Theorem 3.1).

Definition 2.4. The element $\operatorname{det} D \in \mathbb{Z}[[t]]$ is called the Novikov torsion of the pair $(f, v)$ and is denoted by $\tau(f, v)$ or $\tau_{g}$.

Proceeding to the Lefschetz zeta functions, we need to impose yet another restriction on the gradient flow.

Definition 2.5. Let $f: M \rightarrow S^{1}$ be a Morse function on a closed manifold $M$ and $v$ an $f$-gradient. We say that $v$ is of finite dynamics if for every $n \in \mathbb{Z}$ the set of all closed orbits $\gamma$ satisfying $f_{*}([\gamma])=n \in H_{*}\left(S^{1}\right)$ (where $[\gamma] \in H_{1}(M)$ is the homology class of $\gamma$ ) is finite.

For a half-transversal $f$-gradient of finite dynamics we can define the dynamical Lefschetz zeta function of $(-v)$ :

$$
\zeta_{-v}(t)=\exp \left(\sum_{\gamma} \frac{\varepsilon(\gamma)}{m(\gamma)} t^{m(\gamma)}\right),
$$

where the sum is extended over the set of all closed orbits $\gamma$ of $(-v), \varepsilon(\gamma)$ is the Poincaré index of $\gamma$, and $m(\gamma)$ is the multiplicity of $\gamma$. It is clear that $\zeta_{-v}$ is equal to the Lefschetz zeta function of the diffeomorphism $g$ :

$$
\zeta_{g}(t)=\exp \left(\sum_{n \geq 1} \frac{L\left(g^{n}\right)}{n} t^{n}\right),
$$

where $L\left(g^{n}\right)$ is the graded trace of the homomorphism induced by $g$ in the homology.

Now we define the class of gradient flows with which we shall be working in this paper.

Definition 2.6. Let $M$ be a three-dimensional closed manifold, and $f: M \rightarrow S^{1}$ a Morse function without critical points of indices 0 or 3 ; let $v$ be a half-transversal $f$-gradient of finite dynamics. We say that $(f, v)$ is a regular Morse pair.

We shall also work with Morse functions $f: M \rightarrow S^{1}$ on manifolds with boundary. The definition of a regular Morse pair $(f, v)$ is carried over to this setting in an obvious way, with the following modifications:

1) the restriction $f \mid \partial M: \partial M \rightarrow S^{1}$ is required to be a fibration whose monodromy is isotopic to identity;

2) the gradient vector field $v$ is required to be tangent to $\partial M$. Such a gradient is called a gradient of finite dynamics if for every $n \in \mathbb{Z}$ the set of all closed orbits $\gamma$ satisfying $f_{*}([\gamma])=n$ is finite. 
For a regular Morse pair $(f, v)$ on a 3-dimensional manifold with boundary, we define the Novikov complex $\mathcal{N}_{*}(f, v)$ and the Lefschetz zeta function $\zeta_{-v} \in \mathbb{Z}[[t]]$, which counts the closed orbits of $(-v)$ not included in the boundary $\partial M$.

\section{§3. The Novikov complex And the zeta function OF HALF-TRANSVERSAL FLOWS}

The attractive feature of half-transversal flows is that the Novikov boundary operators and the Lefschetz zeta function of the gradient flow are accessible here through calculations with homotopical quantities associated with the monodromy. Let $M$ be a closed 3-manifold and $(f, v)$ a regular Morse pair on $M$. Let $\bar{M}$ denote the infinite cyclic covering of $M$ corresponding to $f$ and $\Delta_{*}(\bar{M})$ the simplicial chain complex of $\bar{M}$. Set $\Lambda=\mathbb{Z}\left[t, t^{-1}\right]$ and $\widehat{\Lambda}=\mathbb{Z}[[t]]\left[t^{-1}\right]=\mathbb{Z}((t))$. Both $\mathcal{N}_{*}(f, v)$ and $\widehat{\Delta}_{*}(\bar{M})=\Delta_{*}(\bar{M}) \otimes \widehat{\Lambda}$ are based free finitely generated chain complexes over $\widehat{\Lambda}$. The next theorem asserts in particular that there is a chain equivalence between them. A usual procedure allows us to associate the torsion with each such equivalence, which is an element in

$$
\operatorname{Wh}(\widehat{\Lambda})=K_{1}(\widehat{\Lambda}) / U
$$

where $U$ is the subgroup of all elements of the form $\pm t^{n}$. The group $\operatorname{Wh}(\widehat{\Lambda})$ is easily identified with the multiplicative group of all power series in $\mathbb{Z}[[t]]$ with first coefficient equal to 1 (see [20, Chapter 13, §4] for the details), so we shall consider the torsions as power series with coefficients in $\mathbb{Z}[[t]]$. The next theorem is our main aim in this section.

Theorem 3.1. Let $M$ be a closed 3-manifold and $(f, v)$ a regular Morse pair on $M$. There is a chain homotopy equivalence

such that

$$
\phi: \mathcal{N}_{*}(f, v) \rightarrow \Delta_{*}(\bar{M}) \underset{\Lambda}{\otimes} \widehat{\Lambda}
$$

$$
\tau(\phi)=\zeta_{-v}
$$

Observe that this theorem implies the isomorphism

$$
H_{*}\left(\mathcal{N}_{*}(f, v)\right) \approx H_{*}(\bar{M}) \underset{\Lambda}{\otimes} \widehat{\Lambda} .
$$

First, we outline the proof. Lift $f: M \rightarrow S^{1}$ to a Morse function $F: \bar{M} \rightarrow \mathbb{R}$. The regular level surface $S \subset M$ (see Definition 2.2) lifts to a regular level surface of $F$, which will be denoted by the same letter $S$. Denote by $S^{-}$the part of $\bar{M}$ lying below $S$ with respect to the function $F$. We shall construct a certain chain complex $\mathcal{Z}_{*}$ that is free over $\mathbb{Z}[t]$ and computes the homology of $S^{-}$. Then we construct an embedding

$$
\mathcal{N}_{*}(f, v) \hookrightarrow \widehat{\mathcal{Z}}_{*}=\mathcal{Z}_{*} \underset{P}{\otimes} \widehat{P}, \quad \text { where } P=\mathbb{Z}[t], \quad \widehat{P}=\mathbb{Z}[[t]],
$$

such that the quotient complex is acyclic and its torsion is equal to the Lefschetz zeta function of $-v$. The schema of the argument resembles that of the papers [12] and [19], however the present case is in a sense simpler, due to a very particular nature of the half-transversal flows.

Proceeding to details, first we return to the cobordism $Y$ obtained from $M$ by cutting along $S$. We have the naturally arising diffeomorphisms $\psi_{+}: \partial_{+} Y \rightarrow S, \psi_{-}: \partial_{-} Y \rightarrow S$. Put

$$
c_{i}=\mathcal{W}^{u n}\left(p_{i}, v\right) \cap \partial_{+} Y .
$$

Replacing $Y$ by a diffeomorphic cobordism if necessary, we may always assume that the circles $c_{i}, 1 \leq i \leq k$, are standardly embedded in $\partial_{+} Y$ as shown in Figure 1. They are therefore a part of the standard cellular decomposition of $\partial_{+} Y$, which consists of $m$ 


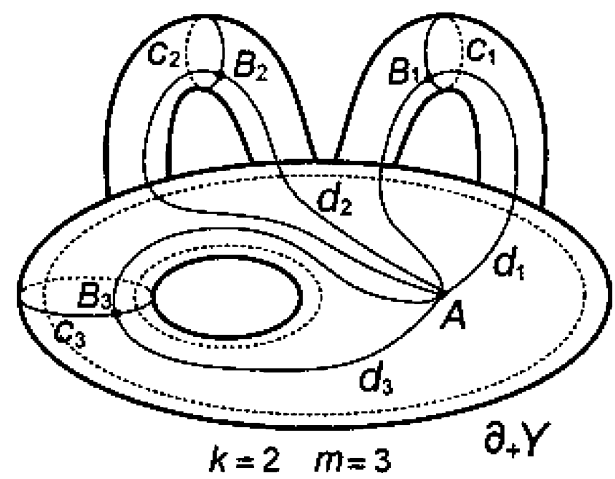

FiguRe 1
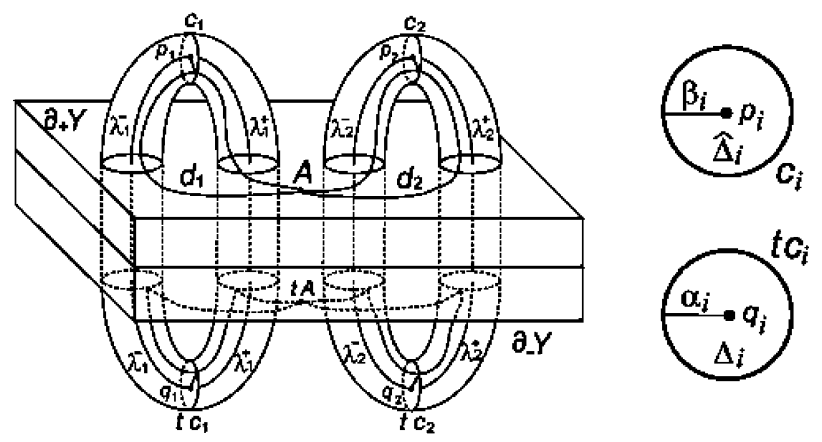

FiguRe 2

disjoint circles $c_{i}$ and $m$ circles $d_{i}$ having a common point $A$. For a subset $X \subset \partial_{+} Y$ we denote by $T X$ the track of $X$, that is,

$$
T X=\{\gamma(x, t ;-v) \mid t \geq 0 \text { and } x \in X\} .
$$

Now we define a filtration $\mathcal{E}^{i}$ in the cobordism $Y$. The term $\mathcal{E}^{0}$ of the filtration contains two points: $A$ and $t A$. The term $\mathcal{E}^{1}$ contains $\mathcal{E}^{0}$ and the following subsets: the circles $d_{i}, c_{i}$, the track $T A$ of the point $A$, the circles

$$
\gamma_{i}=\mathcal{W}^{u n}\left(q_{i}\right) \cup \mathcal{W}^{s t}\left(p_{i}\right) \text { for } i \leq i \leq k,
$$

the $\operatorname{arcs} \alpha_{i}, \beta_{i}$ as shown in Figure 2 and the circles $I c_{i}, I d_{i} \subset \partial_{-} Y$. The term $\mathcal{E}^{2}$ contains $\mathcal{E}^{1}$ and the following subsets: the boundary $\partial Y$ of $Y$, the stable manifolds of the critical points of index 2 and the unstable manifolds of the critical points of index 1 , and the closure of the tracks of $c_{i}$ and $d_{i}$. The term $\mathcal{E}^{3}$ is the entire $Y$. It is not difficult to see that $\mathcal{E}^{i}$ is a cellular filtration of $Y$, that is, the homology of the quotient $\mathcal{E}^{i} / \mathcal{E}^{i-1}$ does not vanish only in degree $i$.

Now we use this filtration to explore the homotopy type of the covering $\bar{M}$. The natural map $Y \rightarrow M$ lifts to an embedding of $Y$ in $\bar{M}$ whose image will be identified with $Y$. The covering $\bar{M}$ is the union of the images of $Y$ under the action of $\mathbb{Z}$ :

$$
\bar{M}=\bigcup_{n \in \mathbb{Z}} t^{n} Y
$$

where $t$ is the downward generator of $\mathbb{Z}$, so that $F(t x)=F(x)-1$ for every $x \in \bar{M}$. The neighbor copies $t^{n} Y$ and $t^{n+1} Y$ intersect by $\partial_{-} t^{n} Y=t^{n} \partial_{-} Y=t^{n+1} \partial_{+} Y=\partial_{+} t^{n+1} Y$. 
Recall from $\$ 2$ that the gradient descent determines a diffeomorphism $g: \partial_{+} Y \rightarrow \partial_{-} Y$. We endow $\partial_{-} Y$ with the cellular decomposition induced from $\partial_{+} Y$ by $g$. Let $h$ be any cellular approximation of the map $\psi_{+} \circ \psi_{-}^{-1}: \partial_{-} Y \rightarrow \partial_{+} Y$. Then $\bar{M}$ has the homotopy type of the space

$$
N=\left(\bigsqcup_{n \in \mathbb{Z}} t^{n} Y\right) / \mathcal{R}
$$

where the equivalence relation $\mathcal{R}$ identifies $\partial_{-} t^{n} Y \approx \partial_{-} Y$ with $\partial_{+} t^{n+1} Y \approx \partial_{+} Y$ via the map $h: \partial_{-} Y \rightarrow \partial_{+} Y$. The space $N$ has a natural free action of $\mathbb{Z}$ and we have a homotopy equivalence $\bar{M} \rightarrow N$ respecting this action. Put

$$
N^{-}=\left(\bigsqcup_{n \in \mathbf{N}} t^{n} Y\right) / \mathcal{R} .
$$

Now we use the filtration $\mathcal{E}$ of $Y$ to construct a filtration of $N^{-}$. Put

$$
\mathcal{F}^{i}=\bigcup_{n \in \mathbf{N}} t^{n} \mathcal{E}^{i}
$$

The filtration $\mathcal{S}_{*}\left(\mathcal{F}^{i}\right)$ of the singular chain complex $\mathcal{S}_{*}\left(N^{-}\right)$of $N^{-}$is cellular and the homology

$$
H_{i}\left(\mathcal{F}^{i} / \mathcal{F}^{i-1}\right)
$$

is a free $P$-module. We describe the generators of this module. We denote the stable manifold of $p_{i}$ by $D\left(p_{i} ; v\right)$. The set $D\left(p_{i} ; v\right) \backslash\left\{p_{i}\right\}$ consists of two arcs, their closures will be denoted by $\lambda_{i}^{+}, \lambda_{i}^{-}$(the signs correspond to the chosen orientations). Put $\lambda_{i}=\lambda_{i}^{+} \cup \lambda_{i}^{-}$. Let $\beta_{i}$ be an arc in $\Delta_{i}$ joining $p_{i}$ and $B_{i}=c_{i} \cap d_{i}$. Similarly, let $\alpha_{i}$ be an arc joining $t A$ with $t B_{i}$. Let $d_{i}^{\prime}$ be the part of $d_{i}$ between $A$ and $B_{i}$ and denote by $\chi_{i}^{+}$the following composition of arcs:

$$
\chi_{i}^{+}=d_{i}^{\prime} \cdot \beta_{i} \cdot \lambda_{i}^{+} \cdot \alpha_{i} \cdot\left(t d_{i}^{\prime}\right)^{-1}, \quad \text { where } 1 \leq i \leq k .
$$

Similarly, set

$$
\chi_{i}^{-}=d_{i}^{\prime} \cdot \beta_{i} \cdot \lambda_{i}^{-} \cdot \alpha_{i} \cdot\left(t d_{i}^{\prime}\right)^{-1}, \quad \text { where } 1 \leq i \leq k .
$$

The fundamental class of $\partial_{+} Y$ modulo the union of $c_{i}$ and $d_{i}$ is denoted by $\omega_{2}$. The fundamental class of $Y$ modulo the subspace $\mathcal{E}^{2}$ is denoted by $\omega_{3}$. Here is the list of free generators of $\mathcal{Z}_{r}=H_{r}\left(\mathcal{F}^{r} / \mathcal{F}^{r-1}\right)$ : as a $\mathbb{Z}[t]$-module,

$$
\begin{array}{rlr}
r=0: & A & \\
r=1: & c_{i}, d_{i} & \text { for } 1 \leq i \leq m=\operatorname{genus}\left(\partial_{+} Y\right) \\
r=2: & \chi_{i}^{+}, \chi_{i}^{-} & \text {for } 1 \leq i \leq k . \\
& \omega_{2}, & \\
& \widehat{\Delta}_{i}, \Delta_{i}, T d_{i} & \text { for } 1 \leq i \leq k, \text { and } \\
& T c_{i}, T d_{i} & \text { for } k+1 \leq i \leq m . \\
r=3: & \omega_{3}=T \omega_{2} . &
\end{array}
$$

Here $\widehat{\Delta}_{i}$ is the unstable manifold of $p_{i}$ in $Y$; we have $\partial \widehat{\Delta}_{i}=c_{i}$, and similarly for $\Delta_{i}$. (By a certain abuse of notation we use the same symbol $c_{i}$ for the cycle and its geometric support; a similar convention applies to the other notation.) Now we shall describe the boundary operators in the adjoining complex

$$
\partial_{r}: \mathcal{Z}_{r} \rightarrow \mathcal{Z}_{r-1}:
$$

$\partial_{1}: \mathcal{Z}_{1} \rightarrow \mathcal{Z}_{0}:$

$$
\partial\left(c_{i}\right)=0=\partial\left(d_{i}\right), \quad \partial\left(\chi_{i}^{+}\right)=\partial\left(\chi_{i}^{-}\right)=\partial(T A)=A-t h(A) .
$$


$\partial_{2}: \mathcal{Z}_{2} \rightarrow \mathcal{Z}_{1}:$

$$
\left.\begin{array}{rl}
\partial\left(\widehat{\Delta}_{i}\right) & =-c_{i} \\
\partial\left(\Delta_{i}\right) & =t h\left(c_{i}\right) \\
\partial\left(T d_{i}\right) & =d_{i}+\lambda_{i}-t h\left(d_{i}\right) \\
\partial\left(T c_{i}\right) & =c_{i}-t h\left(c_{i}\right) \\
\partial\left(T d_{i}\right) & =d_{i}-\operatorname{th}\left(d_{i}\right) \\
\partial\left(\omega_{2}\right) & =0 .
\end{array}\right\} \text { for } k+1 \leq i \leq m, \text { and }
$$

$\partial_{3}: \mathcal{Z}_{3} \rightarrow \mathcal{Z}_{2}:$

$$
\partial\left(\omega_{3}\right)=\omega_{2}-t h\left(\omega_{2}\right) .
$$

The chain complex $\mathcal{Z}_{*}$ is chain equivalent to the simplicial chain complex of $N^{-}$. Any chain equivalence

$$
\xi: \mathcal{Z}_{*} \rightarrow \Delta_{*}\left(N^{-}\right)
$$

has a well-defined torsion $\tau(\xi) \in \mathrm{Wh}(\mathbb{Z}[t])=K_{1}(\mathbb{Z}[t]) /\{ \pm 1\}$. Since the last group vanishes (by the Bass-Heller-Swan theorem), we have $\tau(\xi)=0$, and the torsion of the chain equivalence

$$
\widehat{\xi}: \widehat{\mathcal{Z}}_{*}=\mathcal{Z}_{*} \underset{\mathbb{Z}[t]}{\otimes} \mathbb{Z}[[t]] \rightarrow \Delta_{*}\left(N^{-}\right) \underset{\mathbb{Z}[t]}{\otimes} \mathbb{Z}[[t]]
$$

in the group $K_{1}(\mathbb{Z}[[t]]) /\{ \pm 1\}$ vanishes. To prove our theorem, it suffices therefore to construct a chain equivalence

$$
\mathcal{N}_{*}^{-}=\mathcal{N}_{*}^{-}(f, v) \stackrel{\sigma}{\rightarrow} \widehat{\mathcal{Z}}_{*}
$$

such that $\tau(\sigma)=\zeta_{-v}$. We embed $\mathcal{N}_{*}^{-}$to $\widehat{\mathcal{Z}}_{*}=\mathcal{Z}_{*} \underset{\mathbb{Z}[t]}{\otimes} \mathbb{Z}[[t]]$ and compute its quotient complex. First, we observe that the Novikov complex for our half-transversal flow can be expressed in terms of the monodromy $g$ or its homotopy substitute $h$ :

$$
\partial q_{i}=\sum N\left(q_{i}, p_{j}\right) p_{j}, \text { where } N\left(q_{i}, p_{j}\right)=\sum_{k \in \mathbf{N}} t^{k}\left\langle h^{k}\left(c_{i}\right), c_{j}\right\rangle
$$

here $\langle\cdot, \cdot\rangle$ stands for the pairing in $H_{1}\left(\partial_{+} Y\right)$. Now we make a simple change of the basis 2 in $\mathcal{Z}_{*}$, replacing $\widehat{\Delta}_{i}$ by the element $\widehat{\Delta}_{i}-\Delta$ to be denoted by $T c_{i}$ (in order to emphasize the analogy with the tracks of the circles $d_{i}$ ). By extending the map $T$ by linearity to a homomorphism $H_{1}\left(\partial_{+} Y\right) \rightarrow \mathcal{Z}_{2}$, it is easy to check the following formula:

$$
\partial(T \mu)=\mu-t h(\mu)+\sum_{j}\left\langle\mu, c_{j}\right\rangle \lambda_{j} .
$$

Now we make yet another simple change of the basis, replacing the cycle $\Delta_{i}$ by

$$
\widetilde{\Delta}_{i}=\Delta_{i}-\sum_{j=1}^{\infty} t^{j} T\left(h^{j} c_{i}\right) .
$$

This infinite sum corresponds geometrically to the stable manifold of the critical point $p_{i}$. There is however one essential difference between formula (3.2) and similar formulas for the case of the transversal flows (see, e.g., formula (66) in [19]). Formula (3.2) contains the term $T c_{i}=\widehat{\Delta}_{i}-\Delta_{i}$ and similar ones, which, strictly speaking, are not the geometric traces of cells. An easy computation involving formula (3.1) shows that the homomorphism $\sigma: \mathcal{N}_{*}^{-} \rightarrow \mathcal{Z}_{*}$ defined by

$$
\sigma\left(p_{i}\right)=\lambda_{i}, \quad \sigma\left(q_{i}\right)=\widetilde{\Delta}_{i}
$$

\footnotetext{
${ }^{2} \mathrm{~A}$ change of a basis is said to be simple if the torsion of the transition matrix vanishes in $\mathrm{Wh}(\mathbb{Z}[[t]])=$ $K_{1}(\mathbb{Z}[[t]]) /\{ \pm 1\}$.
} 
is an embedding of chain complexes. The quotient complex $Q_{*}$ is also easily computed; here is the list of free $\mathbb{Z}[[t]]$-generators for $Q_{j}$ :

$$
\begin{array}{ll}
j=0: & A \\
j=1: & T A, c_{i}, d_{i}, \chi_{i}^{+} \\
j=2: & T c_{i}, T d_{i}, T d_{i}^{\prime}, \omega_{2} \\
j=3: & T \omega_{3}
\end{array}
$$

We have $\partial\left(T d_{i}^{\prime}\right)=\chi_{i}^{+}$and

$$
\partial(z)=0, \quad \partial(T z)=1-t h(z)
$$

for every $z$ from the following list:

$$
A, c_{i}, d_{i}, \omega_{2}
$$

After factoring out the chain complex generated by $\chi_{i}^{+}$and $T d_{i}^{\prime}$, we obtain the chain complex of the mapping torus of the map $h$. It is well known that its torsion equals the Lefschetz zeta function of $h$ (see Milnor's classical paper [16]). This completes the proof of Theorem 3.1 .

Remark 3.2. The theorem above is valid also in the case of regular Morse pairs on manifolds with boundary, and the proof is similar.

\section{§4. Novikov torsion and the Alexander polynomial for Knots}

Theorem 3.1 establishes a relationship between two natural geometric objects: the homotopy equivalence $\phi: \mathcal{N}_{*}(f, v) \rightarrow \Delta_{*}(\bar{M}) \otimes \widehat{\Lambda}$ and the Lefschetz zeta function of the flow generated by $v$. For computational purposes it is convenient to reformulate it in another way. Let $(f, v)$ be a regular Morse pair on a 3-manifold $M$ (with or without boundary). Let $\mathbf{F}$ be a field.

Definition 4.1. We say that $(f, v)$ is $\mathbf{F}$-acyclic if

$$
H_{*}(\bar{M}) \underset{\mathbb{Z}\left[t, t^{-1}\right]}{\otimes} \mathbf{F}((t))=0 .
$$

Put $\mathcal{N}_{*}(f, v ; \mathbf{F})=\mathcal{N}_{*}(f, v) \underset{\widehat{\Lambda}}{\otimes} \mathbf{F}((t))$. From Theorem 3.1 it follows that if $(f, v)$ is $\mathbf{F}$-acyclic, then the homology of the complex $\mathcal{N}_{*}(f, v ; \mathbf{F})$ also vanishes. The images of the elements $\tau(f, v), \zeta_{-v}$ in the ring $\mathbf{F}[[t]]$ will be denoted by $\tau^{\mathbf{F}}, \zeta_{-v}^{\mathbf{F}}$. The $\mathbf{F}$-acyclicity condition implies that the torsion of the chain complex

is well defined as an element of

$$
\widehat{\Delta}_{*}^{\mathbf{F}}(\bar{M})=\Delta_{*}(\bar{M}) \underset{\mathbb{Z}\left[t, t^{-1}\right]}{\otimes} \mathbf{F}((t))
$$

$$
\mathrm{Wh}(\mathbf{F}((t))) \approx K_{1}(\mathbf{F}((t))) / U
$$

where $U$ is the subgroup of all elements of the form $\pm t^{n}$. We denote this torsion by $\tau_{M}^{\mathbf{F}}$, omitting in the notation the obvious dependence of this element on the homotopy class of $f$.

Proposition 4.2. Under the assumptions of Theorem 3.1, assume moreover that $(f, v)$ is $\mathbf{F}$-acyclic. Then

$$
\tau^{\mathbf{F}} \cdot \zeta_{-v}^{\mathbf{F}}=\tau_{M}^{\mathbf{F}}
$$


Proof. Tensoring by $\mathbf{F}((t))$ the chain equivalence $\phi$, we obtain a chain equivalence

$$
\phi^{\mathbf{F}}: \mathcal{N}_{*}(f, v ; \mathbf{F}) \rightarrow \widehat{\Delta}^{\mathbf{F}}(\bar{M})
$$

of two acyclic complexes. The torsion of such a chain equivalence equals the quotient of the torsions of the complexes.

Let $K \subset S^{3}$ be an oriented knot, $M=S^{3} \backslash \operatorname{Int} N(K)$, and $\mathbf{F}=\mathbf{Q}$. Let $(f, v)$ be a regular Morse pair on $M$ such that the homotopy class $[f] \in H^{1}(M) \approx\left[M, S^{1}\right] \approx \mathbb{Z}$ is the positive generator of this group. The condition of $\mathbb{Q}$-acyclicity is fulfilled here, so the above proposition is valid. It is well known that in this case the torsion $\tau_{M}$ equals the Alexander polynomial divided by $(1-t)$, and we obtain the following corollary.

Corollary 4.3. Let $K$ be a knot in $S^{3}$, let $M=S^{3} \backslash \operatorname{Int} N(K)$, and let $(f, v)$ be a regular Morse pair on $M$. Let $\tau$ be the Novikov torsion of $(f, v)$. Then

$$
\tau \cdot \zeta_{-v}=\frac{\Delta_{K}}{1-t},
$$

where $\Delta_{K}$ stands for the Alexander polynomial of the knot $K$.

\section{$\S 5$. HeegaArd splitting For sutured manifolds}

The notion of a sutured manifold was introduced by Gabai [4]. See also [22]. In this section, we recall the notation and define the Heegaard splitting for the sutured manifolds [6].

Definition 5.1. A sutured manifold $\left(X, R_{+}, R_{-}\right)$is a compact oriented 3-manifold $X$ with $\partial X$ decomposed into the union along the boundary of two connected surfaces $\widetilde{R}_{+}$and $\widetilde{R}_{-}$oriented so that $\partial \widetilde{R}_{+}=\partial \widetilde{R}_{-}=\gamma$ and $\partial X=\widetilde{R}_{+} \cup \widetilde{R}_{-}$. Let $A(\gamma)$ denote a collection of disjoint annuli comprising a regular neighborhood $\gamma$, and define $R_{ \pm}=\widetilde{R}_{ \pm}-\operatorname{Int} A(\gamma)$. Thus, $\partial X=R_{+} \cup R_{-} \cup A(\gamma)$. We regard $R_{+}$as the set of components of $\partial X-\operatorname{Int} A(\gamma)$ whose normal vectors point out of $X$, and $R_{-}$as those whose normal vectors point into $X$. The symbol $R_{ \pm}$will denote $R_{+}$or $R_{-}$(respectively), while $R(\gamma)$ denotes $R_{+} \cup R_{-}$. If $\partial \widetilde{R}_{+}=\partial \widetilde{R}_{-}=\varnothing$, each component of $\widetilde{R}_{ \pm}=R_{ \pm}$is a closed surface.

Let $L$ be a nonsplit oriented link in a homology 3 -sphere, and $\bar{R}$ a Seifert surface of $L$. Set $R=\bar{R} \cap E(L)\left(E(L)=\operatorname{cl}\left(S^{3}-N(L)\right)\right)$. Let $P$ be a regular neighborhood of $R$ in $E(L)$, then $P$ forms $R \times[-1,1]$, where $R=R \times\{0\}$. We denote by $R_{+}$(respectively, $R_{-}$) $R \times\{1\}$ (respectively, $R \times\{-1\})$, then $\left(P, \dot{R}_{+}, \dot{R}_{-}\right)$may be regarded as a sutured manifold. We call $\left(P, \dot{R}_{+}, \dot{R}_{-}\right)$a product sutured manifold for $R$. Next, let $X=\operatorname{cl}(E(L)-P)$, and let $R_{ \pm}=\dot{R}_{\mp}$; then we may also regard $\left(X, R_{+}, R_{-}\right)$as a sutured manifold. We call $\left(X, R_{+}, R_{-}\right)$the complementary sutured manifold for $R$. In this paper, we call this the sutured manifold for $R$ for short.

Example 5.2. Let $K$ be the trefoil knot in the 3-sphere $S^{3}$ and $R$ the genus 1 Seifert surface as illustrated in Figure 3. The (complementary) sutured manifold for $R$ is homeomorphic to the manifold on the right-hand side of the figure. (Note that the 'outside' of the genus 2 surface is the complementary sutured manifold.)

Definition 5.3. A compression body $W$ is a connected 3-manifold obtained from a compact surface $\partial_{-} W$ by attaching 1 -handles to $\partial_{-} W \times\{1\} \subset \partial_{-} W \times[0,1]$. Dually, a compression body is obtained from a connected surface $\partial_{+} W$ by attaching 2 -handles to $\partial_{+} W \times\{1\} \subset \partial_{+} W \times[0,1]$ and 3 -handles to any spheres thereby created. If $W=$ $\partial_{+} W \times[0,1], W$ is called a trivial compression body. 


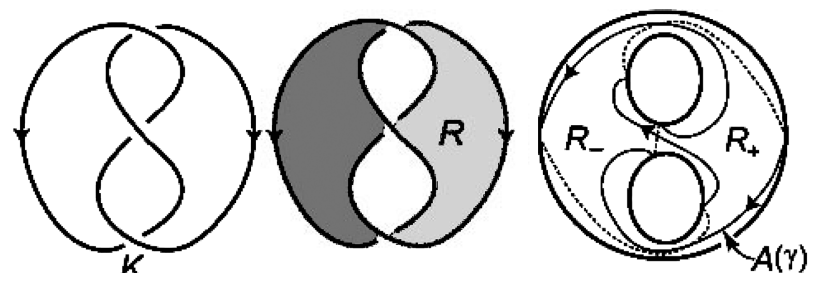

FiguRe 3

We collapse a compression body $W$, so that we may obtain $\partial_{-} W \cup(\operatorname{arcs})$, where the arcs correspond to cores of the attached 1-handles. We call the family of arcs the spine of $W$. We denote by $h(W)$ the number of the attached 1-handles of $W$.

Definition 5.4. We call a pair $\left(W, W^{\prime}\right)$ a Heegaard splitting for a sutured manifold $\left(X, R_{+}, R_{-}\right)$if:

(i) both $W$ and $W^{\prime}$ are compression bodies;

(ii) $W \cup W^{\prime}=X$;

(iii) $W \cap W^{\prime}=\partial_{+} W=\partial_{+} W^{\prime}, \partial_{-} W=R_{+}$and $\partial_{-} W^{\prime}=R_{-}$.

If $\gamma \neq \varnothing$, then $\partial_{-} W$ and $\partial_{-} W^{\prime}$ have boundaries so that $\partial_{(}\left(\partial_{-} W\right) \times[0,1] \cup \partial\left(\partial_{-} W^{\prime}\right) \times$ $[0,1]=A(\gamma)$ and $\partial\left(\partial_{+} W\right)=\partial\left(\partial_{+} W^{\prime}\right)=\gamma$. This case was treated in [6] and [7]. See also [8] for concrete examples. It should be noted that if $R_{+}$is homeomorphic to $R_{-}$, then $h(W)=h\left(W^{\prime}\right)$.

Remark 5.5. This Heegaard splitting corresponds to a circle-valued Morse map $M \rightarrow S^{1}$ for a closed orientable 3-manifold $M$ with $b_{1}(M)>0$ or the complement of a nonsplit link in a homology 3 -sphere $M$. In both cases, we suppose that we have a compact surface $R$ as a representative of $H_{1}(M)$. Then, we obtain the sutured manifold $\left(X, R_{+}, R_{-}\right)$ from $M$ by cutting along $R$. So, we have a Heegaard splitting $\left(W, W^{\prime}\right)$ of $\left(X, R_{+}, R_{-}\right)$ as above. See [9] and 21] for the details.

Definition 5.6. Suppose that $R_{+}$is homeomorphic to $R_{-}$. Set

$$
\begin{aligned}
& h\left(X, R_{+}, R_{-}\right) \\
& \quad=\min \left\{h(W)\left(=h\left(W^{\prime}\right)\right) \mid\left(W, W^{\prime}\right) \text { is a Heegaard splitting for }\left(X, R_{+}, R_{-}\right)\right\} .
\end{aligned}
$$

We call it the handle number of $\left(X, R_{+}, R_{-}\right)$. The Morse-Novikov number $\mathcal{M N}$ of $(M, R)$ or $\left(X, R_{+}, R_{-}\right)$is the minimal possible number of the critical points of the corresponding Morse map.

Remark 5.7. By Corollary 2.8 in [9], we have $\mathcal{M N}(M, R)=2 \times h\left(X, R_{+}, R_{-}\right)$.

Definition 5.8. Suppose that $\left(W, W^{\prime}\right)$ is a Heegaard splitting of a sutured manifold $\left(X, R_{+}, R_{-}\right)$, and let $\lambda$ be a properly embedded arc in $W^{\prime}$ parallel to an arc in $\partial_{+} W^{\prime}$. Here "parallel" means that there is an embedded disk $D$ in $W^{\prime}$ whose boundary is the union of $\lambda$ and an arc in $\partial_{+} W^{\prime}$. Now add a neighborhood of $\lambda$ to $W$ and delete it from $W^{\prime}$. This adds a 1-handle to $W$ (whose core is $\lambda$ ) and also adds a 1-handle to $W^{\prime}$ (whose cocore is a disk in $D$ ). Thus, again we have the Heegaard splitting $\left(\widehat{W}, \widehat{W}^{\prime}\right)$ of $\left(X, R_{+}, R_{-}\right)$where the genus of $\widehat{W}$ (respectively, $\widehat{W}^{\prime}$ ) is one greater than $W$ (respectively, $\left.W^{\prime}\right)$. This process is called a stabilization of $\left(W, W^{\prime}\right)$.

We may regard a compression body $W$ as a sutured manifold $\left(W, R_{+}, R_{-}\right)$, that is, we may assume that $\partial_{+} W=R_{+}$and $\partial_{-} W=R_{-}$. A compression body $W$ has a natural Heegaard splitting: a surface $S$ parallel to $\partial_{+} W$ splits $W$ into two compression bodies, 
at least one of them is trivial. Call this the trivial splitting of $W$. A splitting is said to be standard if it is obtained from the trivial splitting by stabilization. In [23], Scharlemann and Thompson proved the next theorem.

Theorem 5.9 (see [23]). Every Heegaard splitting of a compression body $\left(W, R_{+}, R_{-}\right)$ with $\gamma=\varnothing$ is standard.

Remark 5.10. In [23], two types of trivial splittings, called 'type 1 and 2', were treated. Here we have only to consider the 'type 1 ' trivial splitting.

This theorem induces the following statement. The idea is due to Lei [13].

Theorem 5.11. Any two Heegaard splittings of the same sutured manifold with $\gamma=\varnothing$ have a common stabilization.

Proof. Let $\left(W, W^{\prime}\right)$ and $\left(V, V^{\prime}\right)$ be Heegaard splittings of a sutured manifold $\left(X, R_{+}, R_{-}\right)$ with $\gamma=\varnothing$ such that $\partial_{-} W=R_{+}$and $\partial_{-} V^{\prime}=R_{-}$. Let $\lambda_{W}$ and $\lambda_{V^{\prime}}$ be the spines of $W$ and $V^{\prime}$. Then, a standard general position argument shows that $N\left(\partial_{-} W \cup \lambda_{W}\right) \cap$ $N\left(\partial_{-} V^{\prime} \cup \lambda_{V^{\prime}}\right)=\varnothing$. We denote by $X$ the sutured manifold with $R_{+}=\partial_{+} W$ and $R_{-}=\partial_{+} V^{\prime}$, and let $S$ be a Heegaard splitting surface for $X$. Then $S$ is also a Heegaard splitting surface for $\left(X, R_{+}, R_{-}\right)$. Moreover, $S$ becomes a Heegaard splitting surface for the compression bodies $W^{\prime}=X-\operatorname{Int} N\left(\partial_{-} W \cup \lambda_{W}\right)$ and $V=X-\operatorname{Int} N\left(\partial_{-} V^{\prime} \cup \lambda_{V^{\prime}}\right)$. Hence, the Heegaard splitting surface $S$ is a stabilization of both $\left(W, W^{\prime}\right)$ and $\left(V, V^{\prime}\right)$ by Theorem 5.9.

As in Remark [5.5, if there is a circle-valued Morse map $f: M \rightarrow S^{1}$, we have a Heegaard splitting $\left(W, W^{\prime}\right)$ of the sutured manifold $\left(X, R_{+}, R_{-}\right)$. We also say that $\left(W, W^{\prime}\right)$ is a Heegaard splitting of $M$ or $Y$. Let $\lambda_{W}=\bigcup_{i} \lambda_{W}^{i}$ (respectively, $\lambda_{W^{\prime}}=\bigcup_{i} \lambda_{W^{\prime}}^{i}$ ) be the set of spines of $W$ (respectively, $W^{\prime}$ ).

Definition 5.12. A family $\left(W, W^{\prime}, \lambda_{W}, \lambda_{W^{\prime}}\right)$ is called a symmetric Heegaard splitting of $M$ if it satisfies the following conditions:

(i) $\left(W, W^{\prime}\right)$ is a Heegaard splitting of $M$;

(ii) there is a one-to-one correspondence between the $\operatorname{arcs} \lambda_{W}^{i}$ and $\lambda_{W^{\prime}}^{i}(i=1, \ldots, k)$, and $\partial \lambda_{W}^{i}=\partial \lambda_{W^{\prime}}^{i}$ for each $i$.

Remark 5.13. For a half-transversal gradient flow, we can construct a symmetric Heegaard splitting so that $\bigcup_{i}\left(\lambda_{W}^{i} \cup \lambda_{W^{\prime}}^{i}\right)$ are the circles of the half-transversal flow. Conversely, for every symmetric Heegaard splitting $\mathcal{H}$, there is a homeomorphism $\varphi$ of $Y$ such that $\varphi(\mathcal{H})$ is obtained from a half-transversal gradient flow.

\section{§6. Counting ClOSED orbits}

In this section, we establish a method to count closed orbits by using the idea described in the preceding sections.

Let $R$ be a compact connected manifold, and $g: R \rightarrow R$ a continuous map. Assume that $g$ has only finitely many critical points. The Lefschetz number is defined as follow:

$$
L(g)=\sum_{i=1}^{\ell} \operatorname{ind}\left(x_{i}\right),
$$

where $\operatorname{ind}\left(x_{i}\right)$ is the index of the fixed point $x_{i}$ (see [1]). Let $G_{i}$ be the endomorphism of the homology group $H_{i}(R)$ induced by $g$. Then the Lefschetz fixed point theorem asserts the following:

$$
L(g)=\sum_{i}(-1)^{i} \operatorname{trace}\left(G_{i}: H_{i}(R) \rightarrow H_{i}(R)\right)
$$


Let $K$ be a fibered knot in the 3 -sphere $S^{3}$. Then $K$ has a Seifert surface $R$, and the complement of $K$ is the fiber bundle over $S^{1}$ with fiber $R$. Let $\left(P, \dot{R}_{+}, \dot{R}_{-}\right)$be the product sutured manifold for $R$, and $\left(X, R_{+}, R_{-}\right)$the complementary sutured manifold for $R$. Then $\left(X, R_{+}, R_{-}\right)$has also the structure of a product sutured manifold.

The monodromy $g$ induces a transformation matrix $G_{i}: H_{i}(R) \rightarrow H_{i}(R)$. We call $G_{1}$ the monodromy matrix of the fibered knot $K$. Specifically, we can have the following presentation of $G_{1}$. Let $c_{1}, c_{2}, \ldots, c_{m}, d_{1}, d_{2}, \ldots, d_{m}$ be a symplectic basis of $H_{1}(R)$, where $m$ is the genus of $R$. (See, e.g., [17].) We suppose that $c_{i} \cdot d_{i}=1$ here. Push them off along the normal vector of $R$, and put them on $\dot{R}_{+}$and $\dot{R}_{-}$. Then we may see that they form a basis of $H_{1}\left(\dot{R}_{+}\right)$and $H_{1}\left(\dot{R}_{-}\right)$. Since $R_{ \pm}=\dot{R}_{\mp}$, we may denote the basis of $H_{1}\left(R_{+}\right)$ (respectively, $H_{1}\left(R_{-}\right)$) by $c_{1}^{+}, \ldots, c_{m}^{+}, d_{1}^{+}, \ldots, d_{m}^{+}$(respectively, $\left.c_{1}^{-}, \ldots, c_{m}^{-}, d_{1}^{-}, \ldots, d_{m}^{-}\right)$. By using the product structure of $\left(X, R_{+}, R_{-}\right)$, we push further $c_{1}^{-}, \ldots, c_{m}^{-}, d_{1}^{-}, \ldots, d_{m}^{-}$ into $R_{+}$, and denote their images in $H_{1}\left(R_{+}\right)$by $c_{1}^{\prime}, \ldots, c_{m}^{\prime}, d_{1}^{\prime}, \ldots, d_{m}^{\prime}$. Then

$$
\left(\begin{array}{c}
c_{1}^{\prime} \\
c_{2}^{\prime} \\
\cdot \\
\cdot \\
\cdot \\
d_{m}^{\prime}
\end{array}\right)=G_{1}\left(\begin{array}{c}
c_{1}^{+} \\
c_{2}^{+} \\
\cdot \\
\cdot \\
\cdot \\
d_{m}^{+}
\end{array}\right)
$$

We show an example here.

Example 6.1. Let $K$ be the trefoil knot and $R$ the Seifert surface as shown in Figure 3 , Set $c$ and $d$ as generators of $R$ illustrated in Figure 4 . The upper right-hand figure in Figure 4 shows the sutured manifold $\left(X, R_{+}, R_{-}\right)$for $R$ with $c^{ \pm}, d^{ \pm} \subset R_{ \pm}$. This (complementary) sutured manifold $X$ is a product sutured manifold, that is, $X$ is homeomorphic to $R \times[0,1]$, where $R_{-}=R \times\{0\}$ and $R_{+}=R \times\{1\}$. Then, using this product structure, we can consider a "flow" $\varphi_{s}(s \in[0,1])$ such that $\varphi_{s}(a)=a \times\{s\} \subset R \times\{s\}$ for a subset $a$ in $R_{-} ; \varphi_{s}\left(c^{-}\right)$and $\varphi_{t}\left(d^{-}\right)(s, t \in(0,1),(s \neq t))$ are depicted in the lower left-hand figure in Figure 4, and the lower right-hand figure shows $\varphi_{1}\left(c^{-}\right)$and $\varphi_{1}\left(d^{-}\right)$, denoted by $c^{\prime}$ and $d^{\prime}$. Therefore, we see that

$$
\left(\begin{array}{l}
c^{\prime} \\
d^{\prime}
\end{array}\right)=\left(\begin{array}{c}
d^{+} \\
-c^{+}+d^{+}
\end{array}\right)=\left(\begin{array}{rr}
0 & 1 \\
-1 & 1
\end{array}\right)\left(\begin{array}{l}
c^{+} \\
d^{+}
\end{array}\right)
$$

Thus, we have

$$
G_{1}=\left(\begin{array}{rr}
0 & 1 \\
-1 & 1
\end{array}\right)
$$

In this case, we can observe that $\operatorname{trace}\left(G_{0}: H_{0}(R) \rightarrow H_{0}(R)\right)=1$ and $G_{2}=0$. From (2.4) and (6.1), we have:

$$
\begin{aligned}
\zeta_{g}(t) & =\exp \left(\sum_{k=1}^{\infty} \frac{t^{k}}{k}\left(1-\operatorname{trace} G_{1}^{k}\right)\right) \\
& =\exp \left(\log (1-t)^{-1}+\operatorname{trace}\left(\log \left(I-t \cdot G_{1}\right)\right)\right) \quad(|t|<1) \\
& =\frac{\operatorname{det}\left(I-t \cdot G_{1}\right)}{1-t}=\frac{1-t+t^{2}}{1-t} .
\end{aligned}
$$

Here $I$ is the unit matrix. Note that the Alexander polynomial of the trefoil knot is $1-t+t^{2}$. In general, if a knot $K$ is fibered, the numerator $\operatorname{det}\left(I-t \cdot G_{1}\right)$ equals the Alexander polynomial of $K$. Therefore, we have the following well-known theorem. See, e.g., [16]. 


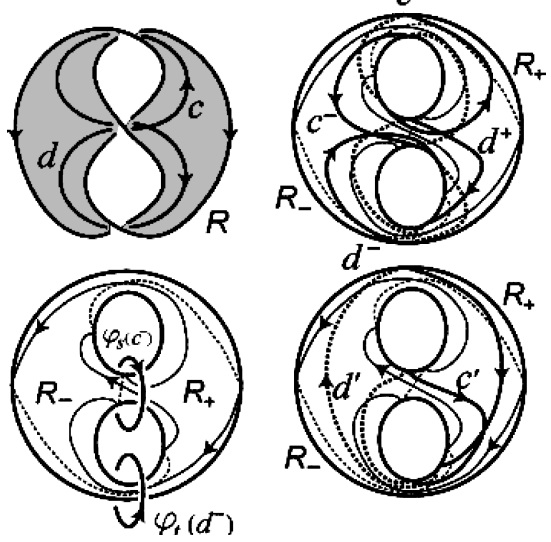

Figure 4

Theorem 6.2 (see [16]). Let $K$ be a fibered knot in $S^{3}$, and let $g$ be the monodromy of $K$. Then

$$
\zeta_{g}(t)=\frac{\Delta_{K}(t)}{1-t}
$$

Here $\Delta_{K}(t)$ is the Alexander polynomial of $K$.

Now, we consider the case of nonfibered knots.

Let $M$ be a compact orientable 3-manifold with $b_{1}(M)>0$. Let $f: M \rightarrow S^{1}$ be a Morse map, and $R$ a regular level surface for $f$. We obtain a sutured manifold $\left(X, R_{+}, R_{-}\right)$from $M$ cutting along $R$. As was pointed out in Remark 5.5 and Definition 5.12 there is a symmetric Heegaard splitting $\left(W, W^{\prime}, \lambda_{W}, \lambda_{W^{\prime}}\right)$ corresponding to $f$. Let $k=h(W)\left(=h\left(W^{\prime}\right)\right)$ be the number of the attached 1-handles of $W$.

In accordance with Definition 2.3, the monodromy $g$ induces the transformation matrix $G_{1}: H_{1}(S) \rightarrow H_{1}(S)$ that can be obtained as follows. We denote the symplectic basis of $H_{1}\left(\partial_{+} W\right)$ (respectively, $\left.H_{1}\left(\partial_{+} W^{\prime}\right)\right)$ by $c_{1}^{+}, \ldots, c_{k}^{+}, c_{k+1}^{+}, \ldots, c_{m}^{+}$, and $d_{1}^{+}, \ldots, d_{k}^{+}$, $d_{k+1}^{+}, \ldots, d_{m}^{+}$(respectively, $c_{1}^{-}, \ldots, c_{k}^{-}, c_{k+1}^{-}, \ldots, c_{m}^{-}$, and $d_{1}^{-}, \ldots, d_{k}^{-}, d_{k+1}^{-}, \ldots, d_{m}^{-}$). Here $c_{j}^{+}$and $d_{j}^{+}$(respectively, $c_{j}^{-}$and $\left.d_{j}^{-}\right)(j=1, \ldots, k)$ are derived from the attached 1-handles of $W$ (respectively, $\left.W^{\prime}\right)$, namely, $c_{j}^{+}$(respectively, $\left.c_{j}^{-}\right)(j=1, \ldots, k)$ is a cocore of the attached 1-handle of $W$ (respectively, $\left.W^{\prime}\right)$ and $d_{j}^{+}$(respectively, $\left.d_{j}^{-}\right)(j=1, \ldots, k$ ) is a 'longitude' corresponding to $c_{j}^{+}$(respectively, $c_{j}^{-}$), so that $c_{j}^{+} \cdot d_{\ell}^{+}=\delta_{j \ell}=c_{j}^{-} \cdot d_{j}^{-}$ $(j, \ell=1, \ldots, m)$, cf. Figure 1. As in the case of a fibered knot, the generators $c_{j}^{+}, d_{j}^{+}$and $c_{j}^{-}, d_{j}^{-}(j=k+1, \ldots, m)$ are obtained from the corresponding generators of $H_{1}(R)$ by using the half-transversal flow associated with $f$, see Figure 5, Let $c_{1}^{\prime}, \ldots, c_{m}^{\prime}, d_{1}^{\prime}, \ldots, d_{m}^{\prime}$ be the images of $c_{1}^{-}, \ldots, c_{m}^{-}, d_{1}^{-}, \ldots, d_{m}^{-}$in $H_{1}\left(\partial_{+} W\right)$. Then

$$
\begin{aligned}
& \left(\begin{array}{lllllllllllll}
c_{1}^{\prime} & \cdots & c_{k}^{\prime} & c_{k+1}^{\prime} & \cdots & c_{m}^{\prime} & d_{1}^{\prime} & \cdots & d_{k}^{\prime} & d_{k+1}^{\prime} & \cdots & d_{m}^{\prime}
\end{array}\right)^{T} \\
& =G_{1}\left(\begin{array}{llllllllllll}
c_{1}^{+} & \cdots & c_{k}^{+} & c_{k+1}^{+} & \cdots & c_{m}^{+} & d_{1}^{+} & \cdots & d_{k}^{+} & d_{k+1}^{+} & \cdots & \left.d_{m}^{+}\right)^{T} .
\end{array}\right.
\end{aligned}
$$

We call $G_{1}$ the monodromy matrix. For $n \geq 1$, we have

$$
\begin{aligned}
& \left(g_{*}^{n}\left(c_{1}^{+}\right) \cdots g_{*}^{n}\left(c_{k}^{+}\right) g_{*}^{n}\left(c_{k+1}^{+}\right) \cdots g_{*}^{n}\left(c_{m}^{+}\right) g_{*}^{n}\left(d_{1}^{+}\right) \cdots g_{*}^{n}\left(d_{k}^{+}\right) g_{*}^{n}\left(d_{k+1}^{+}\right) \cdots g_{*}^{n}\left(d_{m}^{+}\right)\right)^{T} \\
& =G_{1}^{n}\left(\begin{array}{llllllllllll}
c_{1}^{+} & \cdots & c_{k}^{+} & c_{k+1}^{+} & \cdots & c_{m}^{+} & d_{1}^{+} & \cdots & d_{k}^{+} & d_{k+1}^{+} & \cdots & \left.d_{m}^{+}\right)^{T} .
\end{array}\right.
\end{aligned}
$$

Here $(\cdot)^{T}$ stands for the transpose of a matrix. 


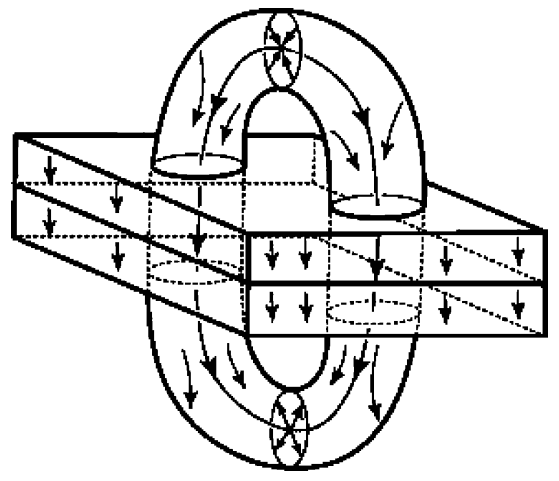

Figure 5

The monodromy $g$ is an orientation preserving diffeomorphism between surfaces; then $G_{1} \in \operatorname{Sp}(2 m, \mathbb{Z})$, in particular $\operatorname{det} G_{1}=1$. Next, $R$ is a closed or once punctured surface in our setting. Whenever $R$ is closed, we have $\operatorname{trace}\left(G_{0}\right)=\operatorname{trace}\left(G_{2}\right)=1$. So, if $|t|$ is sufficiently small, then

$$
\begin{aligned}
\zeta_{g}(t) & =\exp \left(\sum_{k=1}^{\infty} \frac{t^{k}}{k}\left(2-\operatorname{trace} G_{1}^{k}\right)\right) \\
& =\exp \left(\log (1-t)^{-2}+\operatorname{trace}\left(\log \left(I-t \cdot G_{1}\right)\right)\right)=\frac{\operatorname{det}\left(I-t \cdot G_{1}\right)}{(1-t)^{2}} .
\end{aligned}
$$

If $R$ is a once punctured surface, we have

$$
\zeta_{g}(t)=\frac{\operatorname{det}\left(I-t \cdot G_{1}\right)}{1-t}
$$

by the same argument, provided $|t|$ is sufficiently small. Here $I$ stands for the identity matrix.

\section{$\S 7$. Counting Flow Lines}

In this section, we consider counting the gradient flow lines from critical points of index 2 to those of index 1, which are obtained from a circle-valued Morse map $M \rightarrow S^{1}$, in accordance with $\S 2$.

In our setting, where there are only critical points of index 1 and 2 , we can observe the torsion $\tau_{g}(t)$ of the chain complex (2.3) as follows.

As in the preceding sections, we consider only a monodromy matrix obtained from a symmetric Heegaard splitting and a half-transversal flow. The Novikov module $\mathcal{N}_{1}$ (respectively, $\left.\mathcal{N}_{2}\right)$ of the pair $(f, v)$ is generated by $S_{1}(f)=\left\{p_{1}, \ldots, p_{k}\right\}$ (respectively, $\left.S_{2}(f)=\left\{q_{1}, \ldots, q_{k}\right\}\right)$, that is, the center points of the disk bounded by $c_{i}$ (respectively, $\left.t c_{i}\right)$, where $i=1,2, \ldots, k$. See Figures 1 and 2, Therefore the $(i \times(m+j))$-component of the matrix $G_{1}$ stands for the algebraic number of the flow lines between $q_{i}$ and $p_{j}(1 \leq$ $i, j \leq k)$. See Figure 6 for a schematic image. Let $D_{i j}^{(n)}$ be the $(i \times(m+j))$-component of $G_{1}^{n}(1 \leq i, j \leq k)$.

Definition 7.1. We define

$$
\tau_{g}(t)=\operatorname{det}\left(D_{i j}(t)\right), \quad \text { where } D_{i j}(t)=\sum_{n=1}^{\infty}\left(D_{i j}^{(n)} \cdot t^{n-1}\right), \quad 1 \leq i, j \leq k .
$$




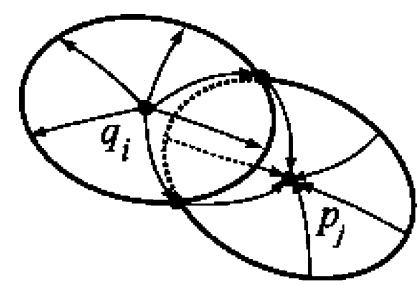

Figure 6
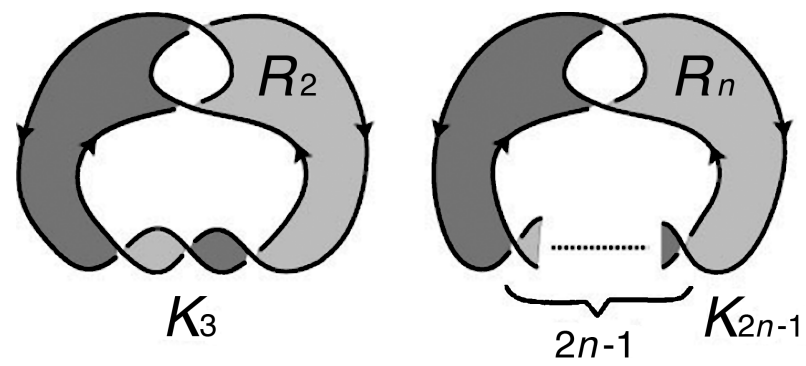

FIGURE 7

If $M$ has no critical points, i.e., $M$ is the fiber bundle over $S^{1}$ with fiber $R$, then $\tau_{g}(t)$ is defined to be 1 .

Taking $|t|$ sufficiently small, we have

$$
\sum_{k=1}^{\infty} G_{1}^{n} \cdot t^{n-1}=G_{1}\left(I-t \cdot G_{1}\right)^{-1} .
$$

Therefore, $D_{i j}(t)$ is the $(i \times(m+j))$-component of $G_{1}\left(I-t \cdot G_{1}\right)^{-1}, 1 \leq i, j \leq k$.

We present examples for $\tau_{g}(t)$ in $\$ 8$.

\section{$\S 8$. Examples}

In this section, we consider twist knots $\mathcal{K}_{2 n-1}(n=1,2,3, \ldots)$. Note that the Alexander polynomial of $\mathcal{K}_{2 n-1}$ is $-n+(2 n-1) t-n t^{2}$. A twist knot has a genus one Seifert surface $R_{n}$ as illustrated in Figure 7. The twist knot $\mathcal{K}_{1}$ is the trefoil knot, it is fibered and treated in Example 6.1. So, we assume that $n \geq 2$.

Let $X_{n}$ be the complement of the knot $\mathcal{K}_{2 n-1}$.

Lemma 8.1. $\mathcal{M N}\left(X_{n}, R_{n}\right)=2$ for any $n, n=2,3, \ldots$

Proof. Let $\lambda$ and $\lambda^{\prime}$ be arcs whose boundaries are in $R_{n}$ as illustrated in Figure 8 , and let $\left(X_{n}, R_{+}, R_{-}\right)$be the sutured manifold for $R_{n}$. Note that $\partial \lambda=\partial \lambda^{\prime}$, and $R_{+}$ (respectively, $R_{-}$) intersects $\lambda$ (respectively, $\lambda^{\prime}$ ) transversely at one point. Then the regular neighborhood of $R_{+} \cup \lambda$ and $R_{-} \cup \lambda^{\prime}$ in $X_{n}$ are compression bodies. Therefore, we only need to show that the sutured manifold cl $\left(\left(X_{n}, R_{+}, R_{-}\right)-\left(N\left(R_{+} \cup \lambda\right) \cup N\left(R_{-} \cup \lambda^{\prime}\right)\right)\right)$, denoted by $\left(\breve{X}_{n}, \breve{R}_{+}, \breve{R}_{-}\right)$, is a product sutured manifold. We consider the case of $\mathcal{K}_{5}$ $(n=3)$; the other cases can be treated by the same method. 

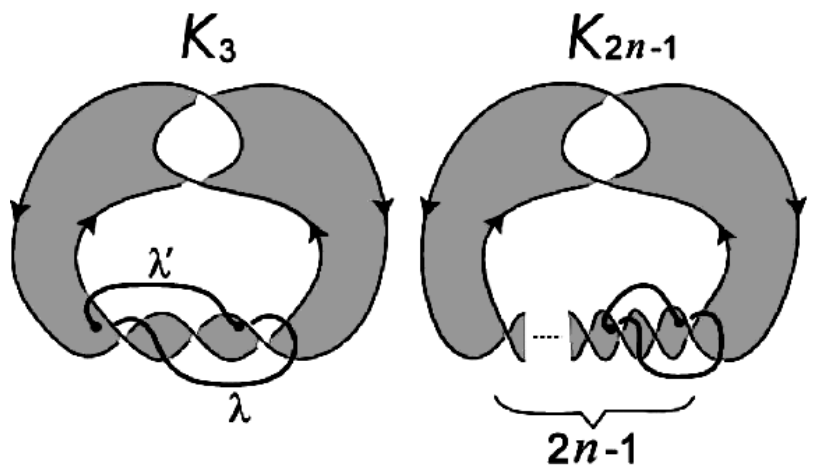

Figure 8
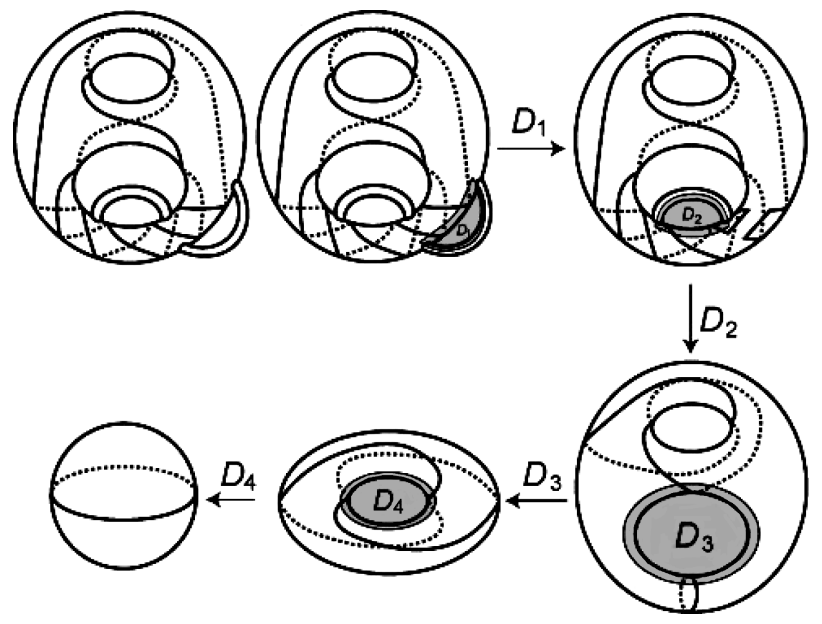

Figure 9

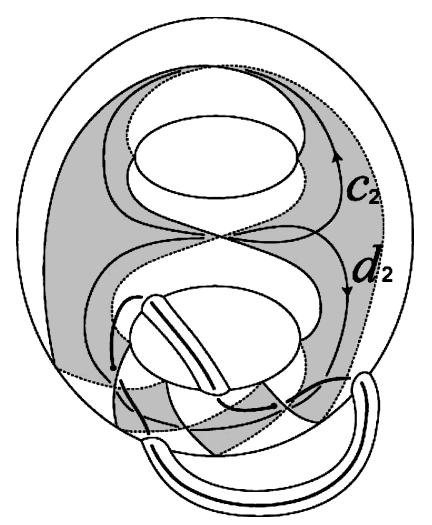

Figure 10 

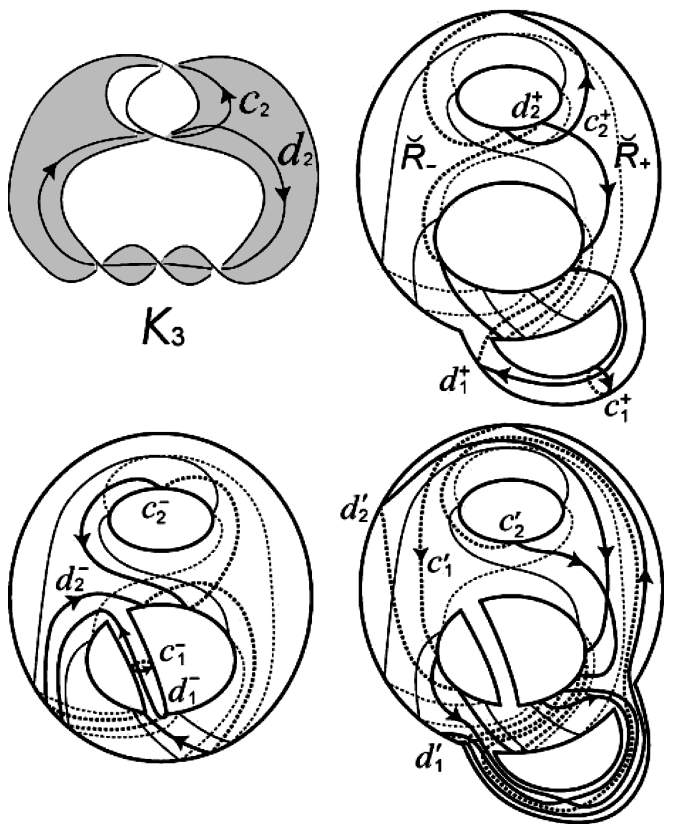

FiguRE 11

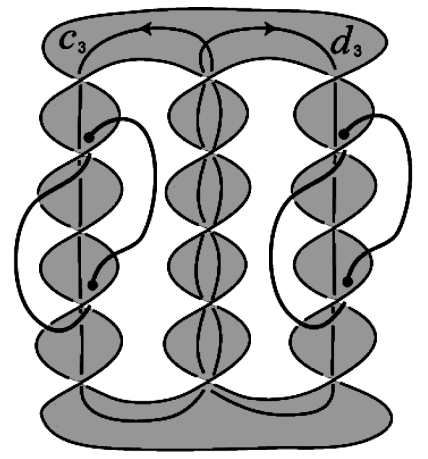

FigURE 12

Let $D_{1}$ be the product disk in $\left(\breve{X}_{3}, \breve{R}_{+}, \breve{R}_{-}\right)$as illustrated in Figure 9 (the shaded part), that is, the disk $D_{1}$ is a properly embedded disk in $\breve{X}_{3}$ such that $\partial D_{1} \cap \breve{R}_{+}$ (respectively, $\partial D_{1} \cap \breve{R}_{-}$) is an arc properly embedded in $\breve{R}_{+}$(respectively, $\breve{R}_{-}$). We decompose $\check{X}_{3}$ along $D_{1}$ and connect the suture naturally, obtaining a new sutured manifold $\left(\breve{X}_{3}^{1}, \breve{R}_{+}^{1}, \breve{R}_{-}^{1}\right)$. This decomposition is called a product decomposition [5]. Similarly, we decompose $\left(\breve{X}_{3}^{1}, \breve{R}_{+}^{1}, \breve{R}_{-}^{1}\right)$ along the product disk $D_{2}$, obtaining a sutured manifold $\left(\breve{X}_{3}^{2}, \breve{R}_{+}^{2}, \breve{R}_{-}^{2}\right)$. See Figure 9] Thus, we have a sequence of product decompositions:

$$
\left(\check{X}_{3}, \check{R}_{+}, \check{R}_{-}\right) \stackrel{D_{1}}{\rightarrow}\left(\check{X}_{3}^{1}, \check{R}_{+}^{1}, \check{R}_{-}^{1}\right) \stackrel{D_{2}}{\rightarrow}\left(\check{X}_{3}^{2}, \breve{R}_{+}^{2}, \breve{R}_{-}^{2}\right) \stackrel{D_{3}}{\rightarrow}\left(\check{X}_{3}^{3}, \breve{R}_{+}^{3}, \breve{R}_{-}^{3}\right) \stackrel{D_{4}}{\rightarrow}\left(\check{X}_{3}^{4}, \breve{R}_{+}^{4}, \breve{R}_{-}^{4}\right),
$$

where $\breve{X}_{3}^{4}$ is homeomorphic to the 3 -ball and both $\breve{R}_{+}^{4}$ and $\breve{R}_{-}^{4}$ are disks. This shows that $\left(\check{X}_{3}, \breve{R}_{+}, \breve{R}_{-}\right)$is a product sutured manifold by [5]. By the same argument, we conclude that $\left(\breve{X}_{n}, \breve{R}_{+}, \breve{R}_{-}\right)$is a product sutured manifold. This completes the proof. 
We denote by $\left(W_{n}, W_{n}^{\prime}, \lambda, \lambda^{\prime}\right)$ the Heegaard splitting of $\left(X_{n}, R_{+}, R_{-}\right)$obtained in the proof of Lemma 8.1 .

Lemma 8.2. The Heegaard splitting $\left(W_{n}, W_{n}^{\prime}, \lambda, \lambda^{\prime}\right)$ is symmetric.

Proof. Since $\partial \lambda=\partial \lambda^{\prime}$ and $\left(\check{X}_{n}, \breve{R}_{+}, \breve{R}_{-}\right)$is a product sutured manifold, we have the claim.

For simplicity, we discuss the case of $\mathcal{K}_{3}(n=2)$ in the next lemma. The general case can be obtained by the same method.

Lemma 8.3. The Heegaard splitting $\left(W_{2}, W_{2}^{\prime}, \lambda, \lambda^{\prime}\right)$ induces a monodromy matrix presented by

$$
G_{1}=\left(\begin{array}{rrrr}
1 & 1 & -2 & -1 \\
0 & 1 & -1 & 0 \\
0 & 0 & 1 & 0 \\
0 & -1 & 0 & 1
\end{array}\right)
$$

Moreover, we have $\zeta_{g}(t)=(1-t)^{3}$ and $\tau_{g}(t)=\frac{-2+3 t-2 t^{2}}{(1-t)^{4}}$.

Proof. Taking a basis $c_{2}, d_{2}$ of $H_{1}(R)$ as illustrated in Figure 10, we get a basis $c_{2}^{+}, d_{2}^{+}$of $H_{1}\left(R_{+}\right)$(respectively, $c_{2}^{-}, d_{2}^{-}$of $H_{1}\left(R_{-}\right)$) as in the upper right-hand figure (respectively, lower left-hand figure) in Figure 11. Note the positions of $\lambda, \lambda^{\prime}$ and $c_{2}, d_{2}$ in Figure 10. Let $\left(\check{X}_{2}, \breve{R}_{+}, \check{R}_{-}\right)$be the sutured manifold $\operatorname{cl}\left(X_{2}, R_{+}, R_{-}\right)-\left(N\left(R_{+} \cup \lambda\right) \cup N\left(R_{-} \cup \lambda^{\prime}\right)\right)$ as in the proof of Lemma 8.1. Here we see that $c_{1}^{+}, c_{2}^{+}, d_{1}^{+}, d_{2}^{+} \subset \breve{R}_{+}$and $c_{1}^{-}, c_{2}^{-}, d_{1}^{-}, d_{2}^{-} \subset$ $\breve{R}_{-}$. Since $\left(\check{X}_{2}, \breve{R}_{+}, \breve{R}_{-}\right)$is a product sutured manifold, we can move $c_{1}^{-}, c_{2}^{-}, d_{1}^{-}, d_{2}^{-}$by a free homotopy from $\breve{R}_{-}$to $\breve{R}_{+}$. We denote their images by $c_{1}^{\prime}, c_{2}^{\prime}, d_{1}^{\prime}, d_{2}^{\prime}$. Then we can see that they sit as in the lower right-hand figure in Figure 11. Hence, $c_{1}^{\prime}=c_{1}^{+}+c_{2}^{+}-2 d_{1}^{+}-d_{2}^{+}$, $c_{2}^{\prime}=c_{2}^{+}-d_{1}^{+}, d_{1}^{\prime}=d_{1}^{+}, d_{2}^{\prime}=-c_{2}^{+}+d_{2}^{+}$. Therefore we have the monodromy matrix $G_{1}$ as in the statement of the lemma, and

$$
\zeta_{g}(t)=\frac{\operatorname{det}\left(I-t \cdot G_{1}\right)}{1-t}=\frac{(1-t)^{4}}{1-t}=(1-t)^{3} .
$$

Note that the convergence radius is 1 .

On the other hand,

$$
G_{1}\left(I-t \cdot G_{1}\right)^{-1}=\left(\begin{array}{cccc}
\frac{1}{(1-t)} & \frac{1}{(1-t)^{3}} & \frac{-2+3 t-2 t^{2}}{(1-t)^{4}} & \frac{-1}{(1-t)^{2}} \\
0 & \frac{1}{1-t} & \frac{-1}{(1-t)^{2}} & 0 \\
0 & 0 & \frac{1}{1-t} & 0 \\
0 & \frac{-1}{(1-t)^{2}} & \frac{t}{(1-t)^{3}} & \frac{1}{1-t}
\end{array}\right) .
$$

Thus, $\tau_{g}(t)=\frac{-2+3 t-2 t^{2}}{(1-t)^{4}}$.

By the same argument, we have the following statement.

Proposition 8.4. The Heegaard splitting $\left(W_{n}, W_{n}^{\prime}, \lambda, \lambda^{\prime}\right)$ induces a monodromy matrix presented by

$$
\left(\begin{array}{rrrr}
1 & 1 & -n & -1 \\
0 & 1 & -1 & 0 \\
0 & 0 & 1 & 0 \\
0 & -1 & 0 & 1
\end{array}\right)
$$

Moreover, we have $\zeta_{g}(t)=(1-t)^{3}$ and $\tau_{g}(t)=\frac{-n+(2 n-1) t-n t^{2}}{(1-t)^{4}}$. 
Example 8.5. Let $K$ be the pretzel knot of type $(5,5,5)$ and consider the symmetric Heegaard splitting associated with Figure 12. Then

$$
G_{1}=\left(\begin{array}{rrrrrr}
1 & 0 & 1 & -5 & -2 & 0 \\
0 & 1 & 0 & -3 & -5 & 1 \\
0 & 0 & 1 & 0 & 1 & 0 \\
0 & 0 & 0 & 1 & 0 & 0 \\
0 & 0 & 0 & 0 & 1 & 0 \\
0 & 0 & 0 & -1 & 0 & 1
\end{array}\right)
$$

Thus, we have $\zeta_{g}(t)=(1-t)^{5}$. Further, $D_{11}(t)=D_{22}(t)=\frac{-5}{(1-t)^{2}}, D_{12}(t)=\frac{-2+3 t}{(1-t)^{3}}$, $D_{21}(t)=\frac{-3+2 t}{(1-t)^{3}}, \tau_{g}(t)=\frac{19-37 t+19 t^{2}}{(1-t)^{6}}$.

\section{ACKNOWLEDGMENT}

This work was initiated when the first and third authors were visiting Institut des Hautes Études Scientifiques in France and continued while the third author was visiting Tokyo University of Agriculture and Technology, supported by Japan Society for the Promotion Science (JSPS). The final version was done while the first author visited Université de Nantes and the second author visited Columbia University, supported by JSPS. They would like to thank these institutions for their hospitality and support.

\section{REFERENCES}

[1] A. Dold, Lectures on algebraic topology, Grundlehren Math. Wiss., Bd. 200, Springer, Berlin, 1972. MR0415602 (54:3685)

[2] S. K. Donaldson, Topological field theories and formulae of Casson and Meng-Taubes, Proc. of the Kirbyfest (Berkeley, CA, 1998), Geom. Topol. Monogr., vol. 2, Geom. Topol. Publ., Coventry, 1999, pp. 87-102. MR.1734402(2001f:57033)

[3] R. Fintushel and R. Stern, Knots, links, and 4-manifolds, Invent. Math. 134 (1998), no. 2, 363-400. MR.1650308 (99j:57033)

[4] D. Gabai, Foliations and the topology of 3-manifolds, J. Differential Geom. 18 (1983), no. 3, 445503. MR 723813(86a:57009)

[5] _ Detecting fibred links in $S^{3}$, Comment. Math. Helv. 61 (1986), no. 4, 519-555. MR870705 (88c:57009)

[6] H. Goda, Heegaard splitting for sutured manifolds and Murasugi sum, Osaka J. Math. 29 (1992), no. 1, 21-40. MR1153177(93d:57014)

[7] - On handle number of Seifert surfaces in $S^{3}$, Osaka J. Math. 30 (1993), no. 1, 63-80. MR 1200821 (93k:57010)

[8] - Circle valued Morse theory for knots and links, Floer Homology, Gauge Theory and Lowdimensional Topology, Clay Math. Proc., vol. 5, Amer. Math. Soc., Providence, RI, 2006, pp. 71-99. MR2249249 (2007e:57007)

[9] H. Goda and A. Pajitnov, Twisted Novikov homology and circle-valued Morse theory for knots and links, Osaka J. Math. 42 (2005), no. 3, 557-572. MR2166722 (2006e:57015)

[10] _ Dynamics of gradient flows in the half-transversal Morse theory, Proc. Japan Acad. Ser. A Math. Sci. 85 (2009), no. 1, 6-10. MR2488751 (2009k:57048)

[11] M. Hutchings and Y.-J. Lee, Circle-valued Morse theory, Reidemeister torsion, and Seiberg-Witten invariants of 3-manifolds, Topology 38 (1999), no. 4, 861-888. MR1679802 (2000i:57055)

[12] _ Circle-valued Morse theory and Reidemeister torsion, Geom. Topol. 3 (1999), 369-396. MR.1716272 (2000h:57063)

[13] F. Lei, On stability of Heegaard splittings, Math. Proc. Cambridge Philos. Soc. 129 (2000), no. 1, 55-57. MR1757777 (2001c:57023)

[14] T. Mark, Torsion, TQFT and Seiberg-Witten invariants of 3-manifolds, Geom. Topol. 6 (2002), 27-58. MR1885588 (2003c:57032)

[15] G. Meng and C. Taubes, $\underline{\mathrm{SW}}=$ Milnor torsion, Math. Res. Lett. 3 (1996), no. 5, 661-674. MR 1418579 (98j:57049)

[16] J. Milnor, Infinite cyclic coverings, Conf. on the Topology of Manifolds (Michigan State Univ., Mich., 1967), Prindle, Weber \& Schmidt, Boston, Mass., 1968, pp. 115-133. MR0242163 (39:3497) 
[17] S. Morita, Geometry of characteristic classes, Transl. Math. Monogr., vol. 199, Iwanami Ser. Modern Math., Amer. Math. Soc., Providence, RI, 2001. MR.1826571 (2002d:57019)

[18] S. P. Novikov, Multivalued functions and functionals. An analogue of the Morse theory, Dokl. Akad. Nauk SSSR 260 (1981), no. 1, 31-35; English transl., Soviet Math. Dokl. 24 (1981), 222226. MR630459(83a:58025)

[19] A. B. Pajitnov, Simple homotopy type of Novikov complex and $\zeta$-function of the gradient flow, Uspekhi Mat. Nauk 54 (1999), no. 1, 117-170; English transl., Russian Math. Surveys 54 (1999), no. 1, 119-169; e-print dg-ga/970614. MR.1706835 (2001m:57041)

[20] Circle-valued Morse theory, de Gruyter Stud. Math., vol. 32, Walter de Gruyter \& Co, Berlin, 2006. MR2319639(2008c:58008)

[21] A. B. Pajitnov, L. Rudolf, and C. Weber, The Morse-Novikov number for knots and links, Algebra i Analiz 13 (2001), no. 3, 105-118; English transl., St. Petersburg Math. J. 13 (2002), no. 3, 417-426. MR.1850189(2002k:57040)

[22] M. Scharlemann, Sutured manifolds and generalized Thurston norms, J. Differential Geom. 29 (1989), no. 3, 557-614. MR992331 (90e:57021)

[23] M. Scharlemann and A. Thompson, Heegaard splittings of (surface) $\times I$ are standard, Math. Ann. 295 (1993), no. 3, 549-564. MR 1204837 (94B:57020)

[24] V. Turaev, A combinatorial formulation for the Seiberg-Witten invariants of 3-manifolds, Math. Res. Lett. 5 (1998), no. 5, 583-598. MR1666856 (2000c:57027)

Department of Mathematics, Tokyo University of Agriculture and Technology, 2-24-16 NaKa-Cho, Koganei, TOKyo 184-8588, Japan

E-mail address: goda@cc.tuat.ac.jp

Department of Mathematical Sciences, Yamagata University, Yamagata 990-8560, Japan

E-mail address: matsuda@sci.kj.yamagata-u.ac.jp

Laboratoire de Mathématiques, Jean-Leray UMR 6629, Université de Nantes, Faculté des Sciences, 2, Rue de la Houssinière, 44072, Nantes, Cedex, France

E-mail address: andrei.pajitnov@univ-nantes.fr

Received 2/MAR/2013

Originally published in English 